\title{
CHARACTERIZATION OF SIX PUTATIVE PHOTOSYSTEM I MUTANTS IN BARLEY
}

by

\author{
ROGER G. HILLER', BIRGER LINDBERG MØLLER \\ and GUNILLA HOYER-HANSEN
}

Department of Physiology, Carlsberg Laboratory, Gamle Carlsberg Vej 10, DK-2500 Copenhagen Valby

IPermanent address: School of Biology, Macquarie University, N. Ryde, Australia 2113

Keywords: Fluorescence induction kinetics, cytochromes, $P 700$, difference spectra, SDSpolyacrylamide gel electrophoresis, viridis- $n^{34}$, viridis- $z b^{63}$, viridis- $h^{15}$, xantha- $q^{75}$, xantha$q^{76}$, viridis $-q^{42}$

Six putative photosystem I mutants in five different nuclear genes of barley have been characterized with respect to their photosynthetic electron transport capabilities, $P 700$, cytochromes, chlorophyll-proteins and other thylakoid polypeptides. Three of these mutants (viridis- $h^{34},-z b^{63}$, and $-h^{15}$ ) have normal photosystem II activities but are wholly or partly deficient in photosystem I electron transport, $P 700$, chlorophyll $a$-protein 1 and its apoprotein. These three mutants also lack low molecular weight polypeptides which are believed to be integral photosystem I iron-sulfur centres. Of the remaining mutants, xantha-q75 and $-q^{76}$, which are allelic, are mainly depleted in photosystem II and the other (viridis- $q^{42}$ ) had about $75 \%$ of the wild-type activity in both photosystems. The data are discussed in relation to the fluorescence induction kinetics and low temperature fluorescence emission spectra of the mutants.

Abbreviations: $\mathrm{Asc}=$ ascorbic acid; $\mathrm{BSA}=$ bovine serum albumin $\mathrm{Chl}=$ chlorophyll; $\mathrm{Cyt}=$ cytochrome; $\mathrm{DCIP}=$ 2,6-dichlorophenolindophenol; $\mathrm{DCMU}=3$-(3,4-dichlorophenyl)-1,1-dimethylurea; $\mathrm{FeCN}=$ potassium fe:ricyanide; $F_{m}=$ maximal fluorescence; $F_{o}=$ initial fluorescence; $F_{s}=$ steady state fluorescence; HEPES $=N$-2-hydroxyethylpiperazine- $N$ '-2-ethanesulfonic acid; $\mathrm{MeV}=$ methyl viologen; $\mathrm{PD}=0$ phenylenediamine; PS = photosystem; SDS-PAGE = sodium dodecyl sulfate polyacrylamide gel electrophoresis; Tricine $=N$-(tris-(hydroxymethyl)-methyl)-glycine; $\quad$ Tris $=$ tris-(hydroxymethyl)aminomethane. 


\section{INTRODUCTION}

A powerful tool for the correlation of thylakoid polypeptides with respect to their function is the use of mutants affecting polypeptide composition $(20,28)$. With the possible exception of the barley chlorophyll $b$-less mutant and the xantha- $f,-g$ and $-h$ mutants in barley blocking steps in chlorophyll $a$ synthesis, relevant mutants have not been sufficiently well characterized to separate the primary lesion from pleiotropic secondary effects. Five photosystem I mutants from maize (21) selected on the basis of their fluorescence induction kinetics have been shown to lack chlorophyll a-protein 1. In addition to the loss of chlorophyll a-protein 1 with its associated $P 700$ activity, three of these mutants were also deficient in chlorophyll $a / b$ proteins. One of these mutants, hcf*E1481 was examined with respect to total thylakoid polypeptide composition (22) and found to be deficient in an additional low molecular weight polypeptide $(18,000 \mathrm{~mol}$. wt.). Whether the mutants lack other polypeptides is not known at present. Photosystem I mutants of Chlamydomonas have been more extensively characterized as to the relationship between $P 700$-chlorophyll a-protein 1, a 70,000 mol. wt. apoprotein, and photosystem I activity (10). Whether there is an obligatory relationship between all these components is still uncertain as is their relationship to the bound iron-sulfur centres known to be an integral part of photosystem I (11). It has recently been shown (24) that a photosystem I mutant from barley (viridis $-n^{34}$ ) is deficient in chlorophyll $a$-protein I and three low molecular weight polypeptides two of which are probably iron-sulfur centres. In this report we have extended the observations on viridis $-n^{34}$ and examined an additional five potential photosystem I mutants of barley which were selected from a group of 42 viridis mutants on the basis of their fluorescence induction kinetics (25).

\section{MATERIALS AND METHODS}

\subsection{Plant material}

Seeds of wild-type barley (Hordeum vulgare cv. Svalöfs Bonus) and the recessive mutants viridis $-h^{15}$, xantha- $q^{75}$, viridis $-n^{34}$, viridis $-q^{42}$, xanth $a-q^{76}$ and viridis $-z b^{63}$ were germinated in vermiculite moistened with tap water. Seedlings were harvested after growing for 7 days at $20^{\circ} \mathrm{C}$ in continuous white light (1700 lux). The mutant seedlings were separated from the wild. type phenotype by their paler green color. All mutants are seedling lethals and are therefore maintained in the heterozygous state $(25,26)$.

\subsection{Chloroplast and thylakoid isolation}

Chloroplasts were prepared by homogenizing precooled leaves in a medium composed of 0.4 M-sucrose, $200 \mathrm{~mm}-\mathrm{NaCl}, 50 \mathrm{~mm}$-Tricine/ $\mathrm{NaOH}, \mathrm{pH}$ 7.9. The homogenate was filtered through two layers of $31 \mu \mathrm{m}$ nylon gauze and centrifuged $5 \mathrm{~min}$ at $4,000 \mathrm{~g}$. Chloroplast pellets to be used for the analysis of thylakoid polypeptide content by SDS-PAGE were resuspended and osmotically shocked in $10 \mathrm{~mm}$ EDTA, 25 mm-HEPES/NaOH, pH 7.5. The thylakoid pellet obtained after centrifugation for $5 \mathrm{~min}$ at $10,000 \mathrm{~g}$ was resuspended in a small volume of $1.90 \mathrm{M}$-sucrose, $5 \mathrm{~mm}$-EDTA, and 25 mM-HEPES, pH 7.5, and the thylakoids purified by the flotation technique described earlier (20). Thylakoids used for the analysis of chlorophyllproteins were subjected to two additional washes with $100 \mathrm{~mm}$-Tris/acetate, $\mathrm{pH}$ 9.2. When chloroplasts were prepared for measurements of photosynthetic electron transfer reactions, $0.5 \%$ BSA was included in the homogenization medium and the chloroplast pellet was resuspended in $50 \mathrm{~mm}$-Tricine, $\mathrm{pH} 8.0,50 \mathrm{~mm}-\mathrm{NaCl}$, and 5 $\mathrm{mm}-\mathrm{MgCl}_{2}$ together with $0.5 \% \mathrm{BSA}$.

\subsection{Chlorophyll determination}

Chlorophyll content of leaves and chloroplasts was determined spectrophotometrically in $80 \%$ acetone (5). For analysis of leaves, only the top 5 $\mathrm{cm}$ was used and this was cut into small pieces and ground in a mortar and pestle in $85 \%$ acetone. Calcium carbonate was present during the grinding to avoid the formation of pheophytin.

\subsection{Photochemical measurements}

\subsubsection{Photosystem I and II measurements}

Spectrophotochemical measurements of photosystem I and II were carried out as described earlier (24) except that methylamine was used as 
an uncoupler instead of gramicidin D. Electron transport was also assayed by use of a Rank Bros. oxygen electrode. Photosystem I plus photosystem II was determined with $\mathrm{H}_{2} \mathrm{O}$ as electron donor and methyl viologen as electron acceptor. Photosystem I electron transport was measured in the presence of DCMU using ascorbate/DCIP as donor and methyl viologen as acceptor. Total electron transport from water to $\mathrm{CO}_{2}$ was estimated from the amount of oxygen evolved upon illumination of small leaf pieces (approx. $20 \mathrm{mg}$ ) in a medium of $100 \mathrm{~mm}$ $\mathrm{NaHCO}_{3}$.

\subsubsection{Cytochromes}

Cytochromes were measured at room temperature as described previously (24). Low temperature spectra of the cytochromes were obtained as described by BOARDMAN and ANDERSON (9).

\subsection{3. $P 700$}

$P 700$ was measured from the ferricyanide oxidized minus ascorbate reduced difference spectrum of chloroplasts dispersed in a Triton X-100 contained buffer (18). The duplicate cuvettes containing the following assay mixture $(2.2 \mathrm{ml})$ : chloroplasts $(150 \mu \mathrm{g}$ chlorophyll), Triton X-100 (11.0 mg) and $42 \mathrm{~mm}$-Tricine, $\mathrm{pH}$ 8.0. The spectrophotometer was operated in the split beam mode and the two cuvettes were measured against each other from 660 to 730 $\mathrm{nm}$ to establish a baseline. Ferricyanide was then added to the reference cuvette to a final concentration of $1 \mathrm{~mm}$ and sodium ascorbate to the measuring cuvette to produce a $1 \mathrm{~mm}$ solution. The two cuvettes were equilibrated for $4 \mathrm{~min}$ before the difference spectrum was recorded. A millimolar extinction coefficient of 64 mequivalents ${ }^{-1} . \mathrm{cm}^{-1}$ was used to convert the observed change in absorbance at 698 minus that at $725 \mathrm{~nm}$ into concentration of $P 700$ (18). Light induced $P 700$ content was assayed as described by ANDERSON et al. (3).

\subsection{Difference spectra of wild-type minus mutants}

Cuvettes containing wild-type and mutant chloroplasts were adjusted to give equal absor- bance $(\mathrm{A}=1.0)$ at the red chlorophyll absorption maximum. The difference spectrum was then recorded from 600 to $750 \mathrm{~nm}$ at both room and liquid nitrogen temperatures using an Aminco DW-2a spectrophotometer. For measurements at $77 \mathrm{~K}$ the chloroplasts were suspended in buffer containing $66 \%(\mathrm{v} / \mathrm{v})$ glycerol.

\subsection{Fluorescence emission spectra}

The fluorescence emission spectra were obtained from whole leaves at liquid nitrogen temperature $(77 \mathrm{~K})$ using an Applied Photophy. sics fibre optics spectrophotometer (25).

\subsection{Sodium dodecyl sulfate polyacrylamide gel electrophoresis}

The proportions of chlorophyll in the different chlorophyll protein complexes and the free chlorophyll band were estimated by scanning cylindrical $8 \%$ SDS-PAGE gels at $670 \mathrm{~nm}$ as previously described (16) except that all gels were run at $4^{\circ} \mathrm{C}$ and at $\mathrm{pH}$ 9.2. An SDS/chlorophyll ratio of $10: 1$ (w/w) was used to solubilize the thylakoid membranes prior to electrophoresis. Increased ratios (up to 20:1 (w/w)) were necessary for proper solubilization of thylakoids from mutants with a low chlorophyll content

Total thylakoid polypeptides were analyzed as described earlier (24).

\section{RESULTS}

\subsection{Partial electron transport reactions}

The mutants used in this study were selected as potential photosystem I mutants from viridis mutants, i.e. mutants originally identified by a reduction in chlorophyll content (25). The mutants contain $10 \%$ to $79 \%$ of the wild type chlorophyll levels as determined by extraction of chlorophyll from leaf pieces (Table I). Lower values obtained for the same mutants by this method compared to the estimates obtained previously from measuring the absorbance of intact leaves are probably due to the relatively large contribution of light scattering in leaves of low chlorophyll content. Exclusive and complete loss of chlorophyll $a$-protein 1 from chloroplasts in leaves having a chlorophyll $a$ to chlorophyll $b$ ratio of 3.0 would result in a chlorophyll $a$ to 
Table I

Chlorophyll content and chlorophyll $a / b$ ratios of wild-type and mutant barley seedlings. Chlorophyll content is expressed as $\mathrm{mg} \cdot \mathrm{g}^{-1}$ fresh weight, and as its percentage relative to wild-type. Data previously obtained (25) for chlorophyll content estimated by absorbance measurements of mutant leaves are also included ( $\%$ *).

\begin{tabular}{lccccccc}
\hline & wild-type & vir- $h 15$ & vir-zb63 & vir- $n^{34}$ & xan- $q^{75}$ & xan- $q^{76}$ & vir- $q^{42}$ \\
\hline chl content & 1.63 & 0.45 & 0.75 & 1.28 & 0.22 & 0.16 & 0.46 \\
$\%$ & 100 & 28 & 46 & 79 & 13 & 10 & 28 \\
$\% *$ & 100 & 45 & 64 & 76 & 29 & 26 & 25 \\
chl $a /$ chl $b$ ratio & 3.1 & 5.2 & 5.2 & 3.0 & 3.0 & 3.0 & 3.1 \\
\hline
\end{tabular}

\section{Table II}

Photosynthetic electron transport in chloroplasts of wild-type and mutant barley.

\begin{tabular}{|c|c|c|c|c|c|c|c|}
\hline & wild-type & $v i r-h 15$ & $v i r-z b 63$ & $v i r-n 34$ & $x a n-q^{75}$ & $x a n-q^{76}$ & vir-q $q^{42}$ \\
\hline Oxygen electrode & & \multicolumn{6}{|c|}{$\mu$ moles $\mathrm{O}_{2}$ consumed $\cdot \mathrm{hr}^{-1} \cdot \mathrm{mg}^{-1}$ chlorophyll } \\
\hline $\begin{array}{l}\mathrm{PS} \mathrm{I}+\mathrm{PS} \mathrm{II} \\
\left(\mathrm{H}_{2} \mathrm{O} \rightarrow \mathrm{MeV}\right)\end{array}$ & 105 & 9 & 0 & 21 & 0 & 0 & 32 \\
\hline $\begin{array}{l}\text { PS I } \\
(\mathrm{Asc} / \mathrm{DCIP} \rightarrow \mathrm{MeV})\end{array}$ & 185 & 44 & 0 & 64 & 110 & 165 & 134 \\
\hline Spectrophotometric & & \multicolumn{6}{|c|}{$\mu$ moles of reduced acceptor $\cdot \mathrm{hr}^{-1} \cdot \mathrm{mg}^{-1}$ chlorophyll } \\
\hline $\begin{array}{l}\mathrm{PSI}+\mathrm{PS} \mathrm{II} \\
\left(\mathrm{H}_{2} \mathrm{O} \rightarrow \mathrm{FeCN}\right)\end{array}$ & 359 & 66 & 56 & 207 & - & - & 205 \\
\hline $\begin{array}{l}\mathrm{PS} \mathrm{II} \\
\left(\mathrm{H}_{2} \mathrm{O} \rightarrow \mathrm{Pd} / \mathrm{FeCN}\right)\end{array}$ & 541 & 454 & 590 & 736 & 31 & 36 & 402 \\
\hline
\end{tabular}

Table III

$P 700$ content of wild-type and mutant barley thylakoids expresses as molar ratios of total chlorophyll to $P 700$.

\begin{tabular}{llrrrrrr}
\hline & wild-type & vir- $h^{15}$ & vir-zb63 & vir-n $n^{34}$ & xan-q75 & xan-q76 & vir-q42 \\
\hline Chemical chl/P700 & 323 & $>10,000$ & $>10,000$ & 2,130 & 4,150 & 6,070 & 406 \\
Photochemical chl/P700 & 324 & $>15,000$ & $>15,000$ & 3,520 & - & - & - \\
& 416 & - & - & - & - & - & 840 \\
\hline
\end{tabular}

\section{Table IV}

Cytochrome content of wild-type and mutant barley thylakoids. Results are expressed as the molar ratio of total chlorophyll to cytochrome.

\begin{tabular}{lccccccc}
\hline & wild-type & vir- $h^{15}$ & vir-zb & vir-n34 & xan-q75 & xan-q $q^{76}$ & vir-q42 \\
\hline chl/cyt $f$ & 1,220 & 3,750 & 6,280 & 960 & 8,400 & 8,790 & 1,260 \\
chl/cyt $b_{559}$ HP. & 496 & 1,300 & 350 & 320 & 14,000 & $\infty$ & 862 \\
chl/cyt $b_{6}$ & 275 & 283 & 2031 & 358 & 442 & 650 & 360 \\
\hline
\end{tabular}

IThis value is predominantly due to cytochrome $b 559$ low potential - see text. 
Table V

Chlorophyll-proteins of wild-type and mutant barley. Results are expressed as a percentage of the total chlorophyll content in the different chlorophyll containing bands in the gel.

\begin{tabular}{|c|c|c|c|c|c|c|c|}
\hline $\begin{array}{l}\text { Chlorophyll } \\
\text { bands }\end{array}$ & wild-type & $v i r-h^{15}$ & $v i r-z b^{63}$ & vir $-n^{34}$ & $x a n-q^{75}$ & $x a n-q^{76}$ & vir- $q^{42}$ \\
\hline $\begin{array}{l}\mathrm{Chl}_{a}-\mathrm{Pl} \\
\mathrm{Chl}_{a / b}-\mathrm{Pl}+\end{array}$ & 24.1 & 0 & 0 & 9.9 & 2.7 & 2.3 & 17.8 \\
\hline $\begin{array}{l}\mathrm{Chl}_{a / b}-\mathrm{P} 2+ \\
\mathrm{Chl}_{a}-\mathrm{P} 2+ \\
\mathrm{Chl}_{a}-\mathrm{P} 3\end{array}$ & 63.8 & 77.2 & 86.0 & 82.6 & 68.2 & 71.1 & 66.4 \\
\hline Free chl & 12.1 & 22.8 & 14.0 & 7.5 & 29.1 & 26.4 & 15.8 \\
\hline
\end{tabular}

\section{Table VI}

Chlorophyll-proteins of wild-type and mutant barley thylakoids after extended electrophoresis. Results are expressed as a percentage of the total chlorophyll content in the different chlorophyll containing bands in the gel.

\begin{tabular}{lccc}
\hline $\begin{array}{l}\text { Chlorophyll } \\
\text { bands }\end{array}$ & wild-type & vir-h15 & vir-zb63 \\
\hline $\mathrm{Chl}_{a}-\mathrm{Pl}$ & 22.1 & 0 & 0 \\
$\mathrm{Chl}_{a} / b-\mathrm{P2}{ }^{*}$ & 12.6 & 11.1 & 14.5 \\
$\mathrm{Chl}_{a}-\mathrm{P} 3+\mathrm{Chl}_{a}-\mathrm{P} 2$ & 11.0 & 24.2 & 28.9 \\
$\mathrm{Chl}_{a / b}-\mathrm{P} 2$ & 36.7 & 27.8 & 30.5 \\
Free chl & 17.6 & 36.9 & 26.0 \\
\hline
\end{tabular}

chlorophyll $b$ ratio of 1.8 and a chlorophyll content per leaf of approx. $70 \%$ of that of the wild-type. With the exception of the mutant viridis $-n^{34}$, exclusive loss of chlorophyll $a$ protein 1 can not explain the observed reduction in chlorophyll content nor the observed chlorophyll $a$ to chlorophyll $b$ ratios.

The mutants studied are all known to be lethal at the seedling stage $(25,26)$ when grown under field conditions. Localization of the defect may be deduced from measurements of partial electron transport reactions in chloroplasts obtained from seedlings. Results of such studies are presented in Table II. All the mutants show a substantial decrease in the rate of electron transport from water to methyl viologen when this is expressed on a chlorophyll basis. Only in viridis- $z b^{63}$ does this result reflect a complete absence of electron transport through photosys- tem I. However, in mutants viridis $-n^{34}$ and $-h^{15}$ there is a significant reduction in the rate of photosystem I electron transport. The complete absence of total photosynthetic electron transport in the mutants xantha- $q^{75}$ and $-q^{76}$ is surprisingly due to an almost complete absence of photosystem II whereas PSI is only slightly reduced. The mutant viridis $-q^{42}$ has a substantial electron transport capacity in both photosystem I and II whereas measurement of oxygen evolution by leaf pieces using $\mathrm{CO}_{2}$ as electron acceptor showed an inhibition of $85 \%$ compared to the value in leaves of wild-type. When this is expressed on a chlorophyll basis, however, the degree of inhibition is only $50 \%$.

\section{2. $P 700$ content}

The $P 700$ content of wild-type and mutants was determined to see if there is a correlation between the presence of the reaction centre and the photosystem I driven electron transport (Table III). Only two of the mutants appear to be completely deficient in $P 700$, namely viridis $-h^{15}$ and $-z b^{63}$. The mutants viridis $-n^{34}$, xantha $-q^{75}$ and $-q^{76}$ have approx. $10 \%$ of the wild-type level of $P 700$ whereas their photosystem I activities (Table II) vary between 30 and $90 \%$ of the wildtype level. The mutant viridis $-q^{42}$ seems to have close to normal levels of $P 700$ and about $70 \%$ of wild-type photosystem I activity. As usually observed, the photochemically determined chlorophyll $/ P 700$ values are slightly greater than those determined chemically but follow the same general pattern. 


\subsection{Cytochromes}

The cytochrome content of the photosystem I mutant viridis- $n^{34}$ was previously shown to be similar to that of wild-type (24). It was therefore of interest to examine whether this was also true of the photosystem I mutants viridis $-z b^{63}$ and $-h^{15}$. As shown in Table IV both mutants have a decreased content of cytochrome $f$ but apparently normal content of cytochrome $b_{6}$, the other cytochrome associated with photosystem I. However, examination of the dithionite minus hydroquinone spectrum of mutant viridis $-z b^{63}$ indicated an absorption maximum of $559 \mathrm{~nm}$ rather than the usual value of $562 \mathrm{~nm}$ for cytochrome $b_{6}$. Calculation of the content of low potential cytochrome $b_{559}$ relative to that of cytochrome $b_{563}$ showed that the latter was only $30 \%$ of that expected. (Ratio of cytochrome $b_{563}$ to cytochrome $b_{559}$ of 0.6 versus 2.0 for wildtype.) A difference spectrum of dithionite treated chloroplasts of wild-type minus viridis $-z b^{63}$ at $77 \mathrm{~K}$ confirmed this deficiency of cyt $b_{563}$ in addition to the relative absence of cytochrome $f$ (Table IV). Mutants xantha- $q^{75}$ and $-q^{76}$ which are more properly described as photosystem II deficient mutants (Table II) lack cytochrome $b 559$ high potential which is associated with photosystem II. They also, however, have only trace amounts of cytochrome $f$.

\subsection{Thylakoid polypeptides}

The chlorophyll contents and chlorophyll $a / b$ ratios (Table I) indicate that each mutation may have multiple effects on the chlorophyll-protein content. This was investigated by determining the proportion of the amount of chlorophyll associated with each of the principal chlorophyllproteins after solubilization with SDS. Initially, a brief electrophoresis was used to obtain clear separation of chlorophyll $a$-protein 1 from other chlorophyll-proteins together with a minimum of free chlorophyll formation (Table V). There appears to be a complete absence of chlorophyll $a$-protein 1 in both viridis $-2 b^{63}$ and $-h^{15}$ and only about $10 \%$ of the wild-type level in mutants xantha- $q^{75}$ and $-q^{76}$. These amounts of chlorophyll $a$-protein 1 correlate well with the $P 700$ content of the mutants. This also applies to mutant viridis $-q^{42}$ (Table III). The proportion of chlorophyll $a$-protein 1 in viridis $-n^{34}$ is somewhat greater than would be expected from a direct correlation with its reaction centre content but is consistent with the measured photosystem I electron transport rate (Table II).

The chlorophyll content of viridis $-n^{34}$ is so high that visual selection of the mutant leaves from those of the heterozygote and the wild-type is difficult. This may result in some contamination of the mutant chloroplasts with those from normal leaves. To check this possibility, chloroplasts were isolated from single leaves which were indisputably mutant and wild-type, respectively. It was found that the mean chlorophyll $a$ protein 1 content of four mutant leaves was $7.3 \%$ and that of the wild-type $21.1 \%$ expressed as a percentage of the total chlorophyll in the gel. We therefore conclude that the results reported for the large scale chloroplast isolations from viridis $-n^{34}$ are representative of the mutant alone.

The apparent loss of chlorophyll $a$-protein 1 from mutants viridis $-h^{15}$ and $-z b^{63}$ is accompanied by an increased overall chlorophyll $a / b$ ratio. This can only be accommodated by an increase in the proportion of some other chlorophyll $a$-protein and/or a decrease in chlorophyll $a / b$-protein 2 . To investigate this matter, the electrophoretic separation was extended until adequate resolution of chlorophyll $a$ proteins 2 and 3 from chlorophyll $a / b$-protein 2 was achieved (Table VI). Although this extended electrophoretic separation was accompanied by a substantial increase in the proportion of free

Figure 1. Thylakoid polypeptide pattern of wild-type and mutant barley.

The same amount of chlorophyll was applied to each slot and the separation was carried out at $19{ }^{\circ} \mathrm{C}$ on an 11-15\% SDS-polyacrylamide gradient gel. The position of the green bands observed corresponding to chl $a / b-P I$ and $\mathrm{chl}_{\mathrm{a} / \mathrm{b}}-\mathrm{P} 2$ were marked by the use of metal wires before the gel was fixed (right track). The polypeptide composition of photosystem I particles was analyzed for reference purposes. The molecular weights assigned to the polypeptides in the low molecular weight range are inaccurate and only given as a guide. The nomenclature system used for the chlorophyll-proteins is defined in (20). 
R. G. HitLer et al.: Photosystem I mutants in barley

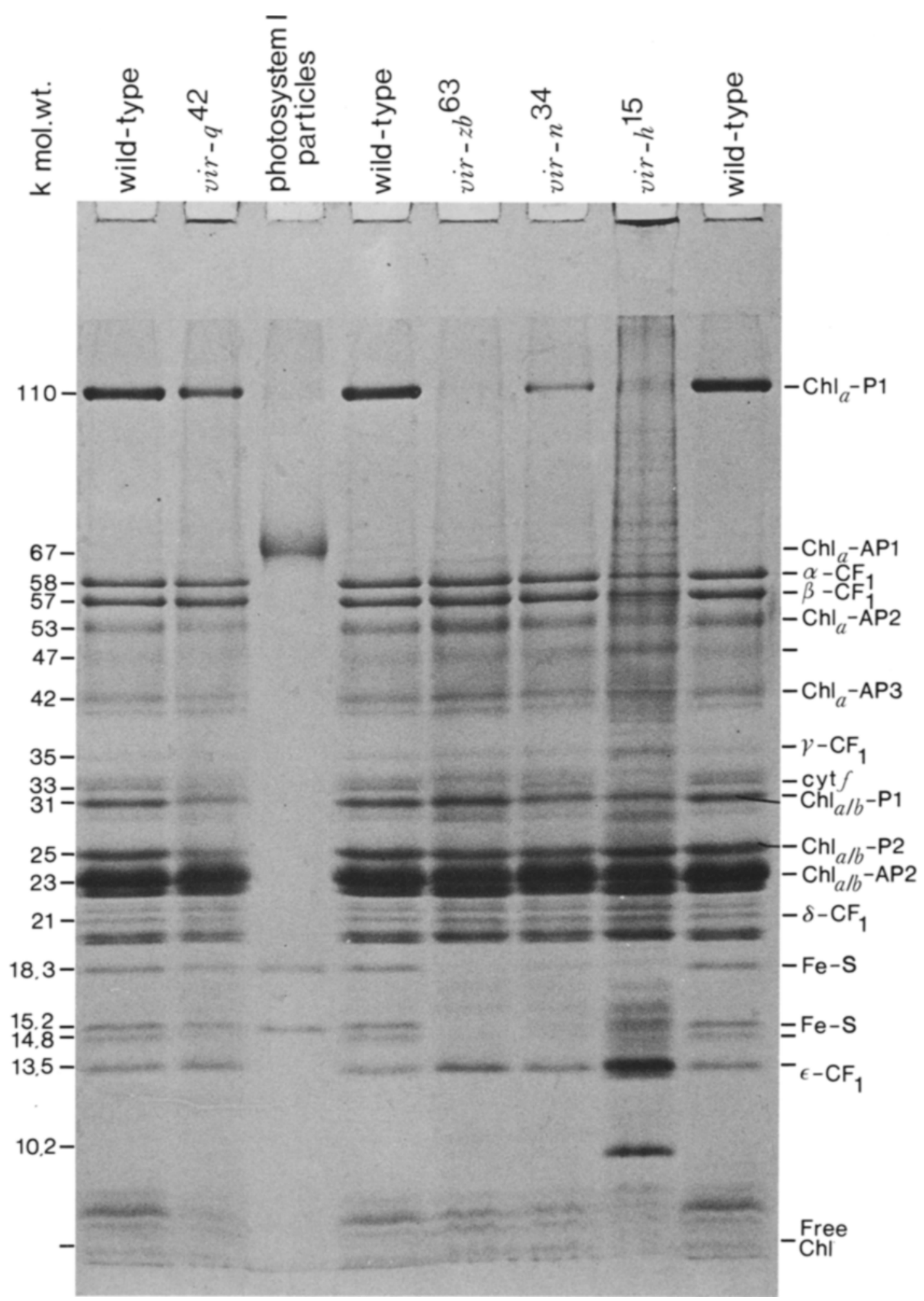




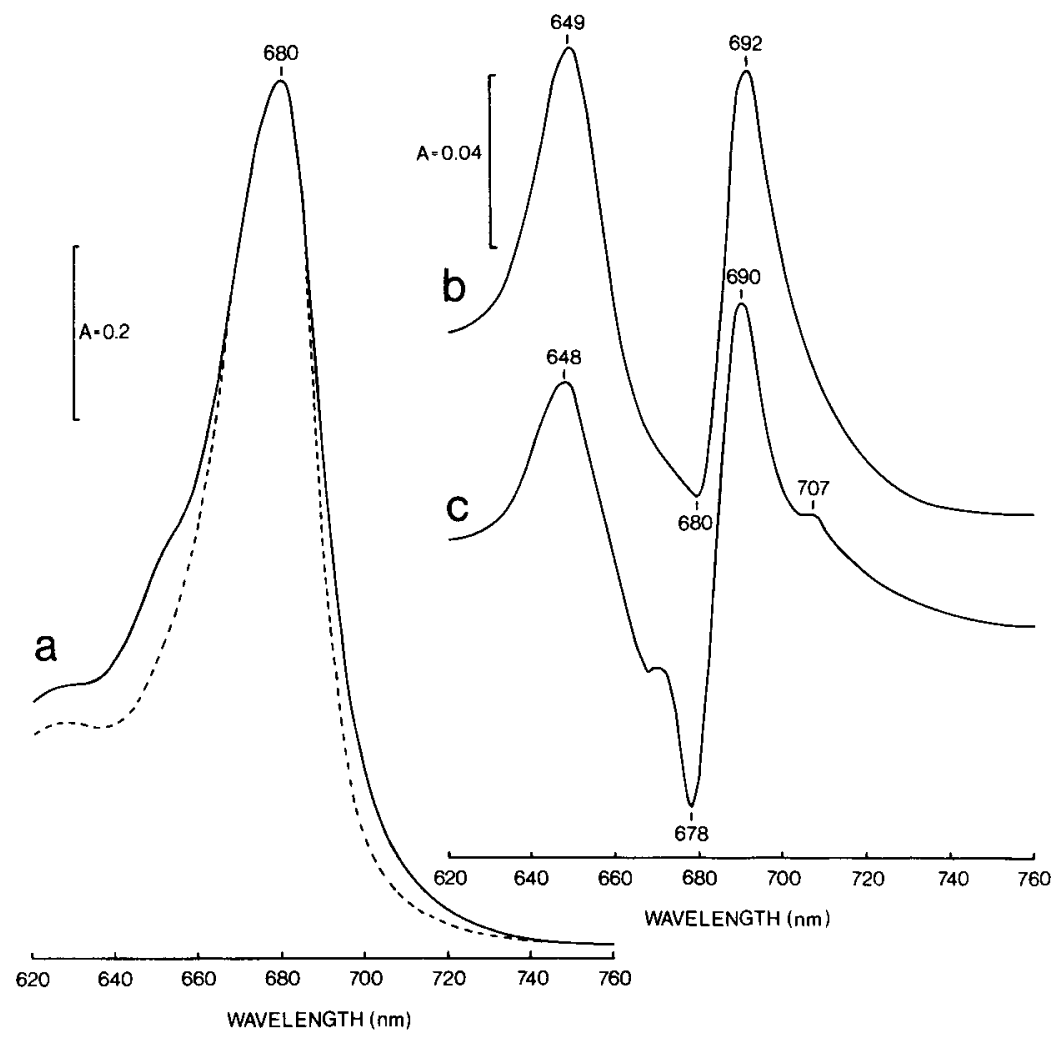

Figure 2. Absorbance and difference spectra of wild-type and viridis-z $z b^{63}$.

a) Absorbance spectra of wild-type (-) and viridis-z $b^{63}(\ldots-\ldots)$. b) Difference spectrum recorded at room temperature. c) Difference spectrum recorded at liquid nitrogen temperature.

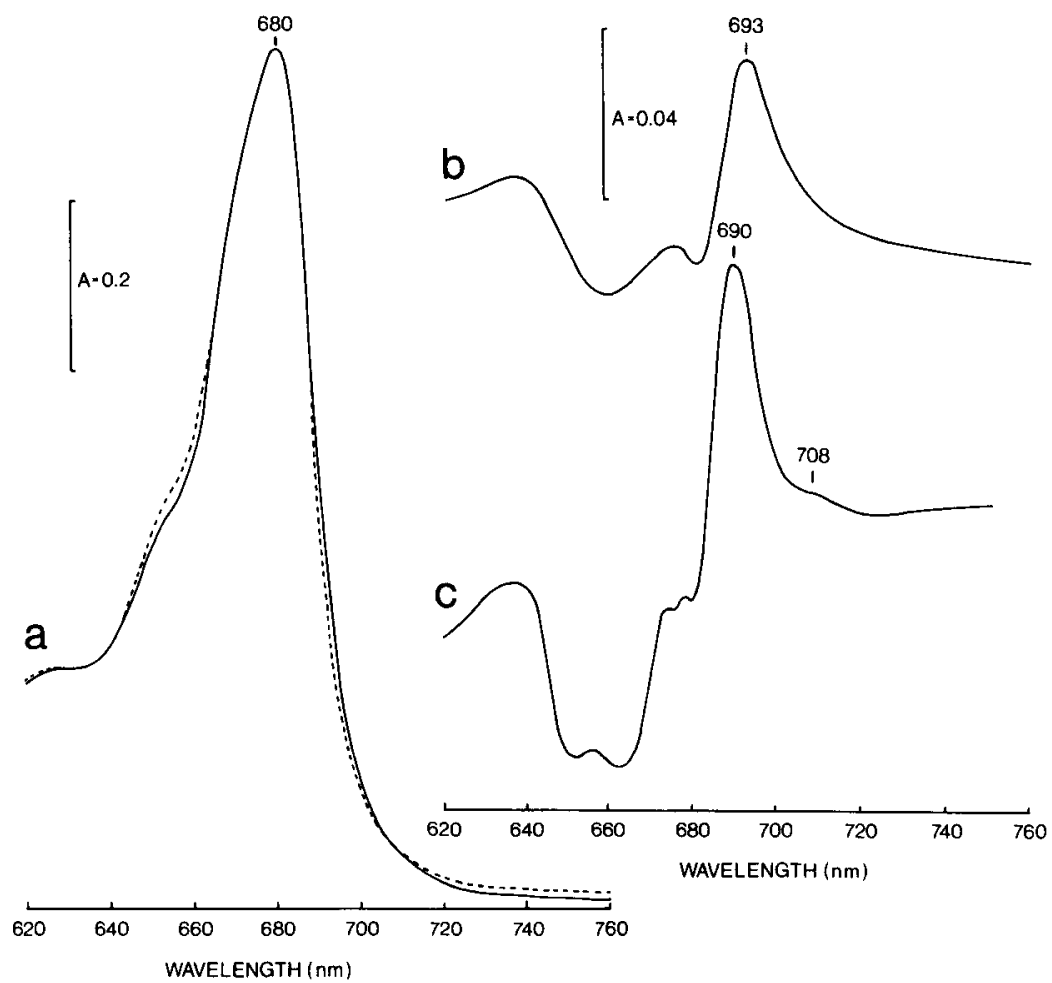

Figure 3. Absorbance and difference spectra of wild-type and viridis $-n^{34}$.

a) Absorbance spectra of wild-type (-) and viridis- $n^{34}(\ldots \ldots)$ b) Difference spectrum recorded at room temperature. c) Difference spectrum recorded at liquid nitrogen temperature. 


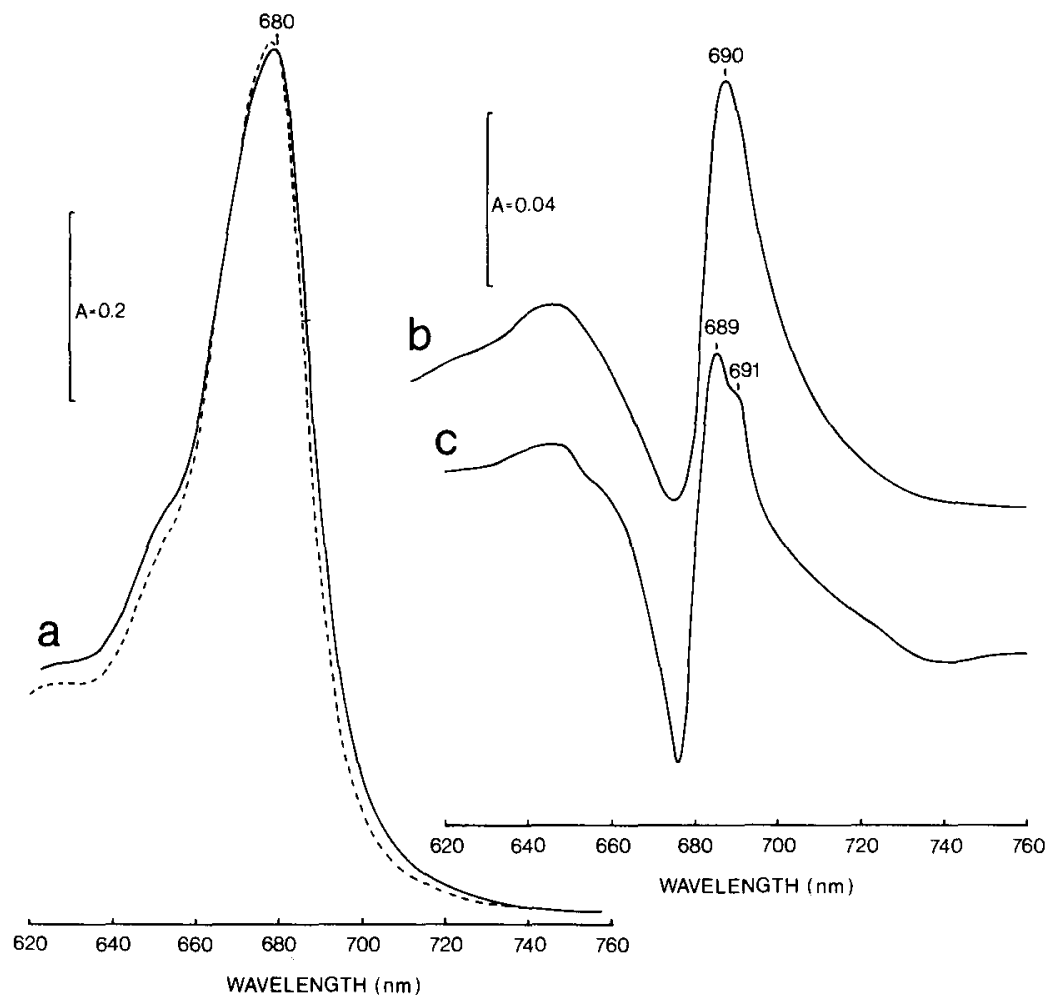

Figure 4. Absorbance and difference spectra of wild-type and viridis- $q^{42}$.

a) Absorbance spectra of wild-type (-) and viridis- 42 (-.-..). b) Difference spectrum recorded at room temperature. c) Difference spectrum recorded at liquid nitrogen temperature.

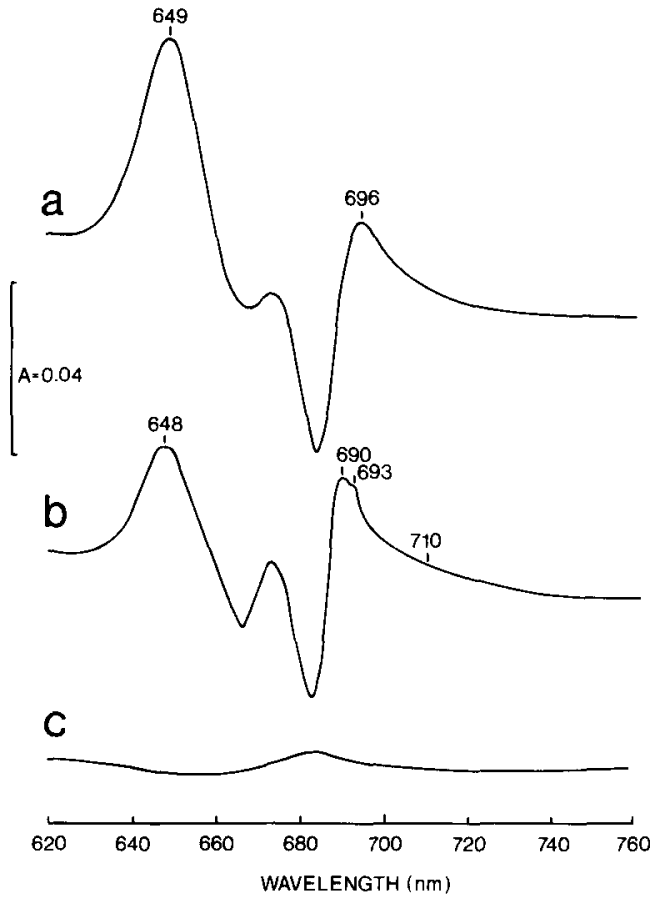

chlorophyll, a remarkable increase of the proportion of the total chlorophyll in chlorophyll $a$ proteins 2 and 3 compared to that in the wildtype was observed.

Total SDS-PAGE thylakoid polypeptide patterns of four of the mutants discussed above are shown in Figure 1. The levels of chlorophyll $a$ protein 1 observed on the gels correspond with the chlorophyll $a$-protein 1 amounts detected on the cylindrical gels. A very faint Coomassie blue stained band at the position of chlorophyll $a$ protein 1 in viridis $-2 b^{63}$ and $-h^{15}$ may represent a comigrating polypeptide or a residual amount of

Figure 5. Difference spectrum of mutants viridis- $q^{42}$ minus viridis $-z b 63$

a) Difference spectrum recorded at room temperature. b) Difference spectrum recorded at liquid nitrogen temperature. c) Control: wild-type minus wild-type, difference spectrum recorded at room temperature. 
chlorophyll $a$-protein 1 . It should be noted that none of the mutants showed an increase in polypeptide content of the $70,000 \mathrm{mw}$ region of the gels. Such an increase might have been expected if the lesion resulted in a decreased stability of chlorophyll $a$-protein I to SDS. The mutant viridis $-n^{34}$ has a reduced amount of three low molecular weight polypeptides (24) two of which are believed to be polypeptides of the ironsulfur centres known to be an integral part of photosystem I. The two photosystem I mutants viridis $-z b^{63}$ and $-h^{15}$ also lack those same low molecular weight polypeptides. In other respects, the polypeptide composition of all six mutants appears normal with the exception of mutants xantha $-q^{75}$ and $-q^{76}$ which are possibly deficient in chlorophyll $a$-proteins 2 and/or 3 (data not shown). Two additional low molecular weight polypeptides (mol. wt. 13,100 and 10,200) in these two mutants and in viridis $-h^{15}$ are believed to be of nuclear origin (19).

In the mutants viridis $-h^{15}$, and $-z b^{63}$, substantial changes in the content of chlorophyll-proteins migrating between chlorophyll $a / b$-protein 2 and its oligomer chlorophyll $a / b$-protein $2^{*}$ were observed after a brief electrophoretic separation. The apoproteins of these components have not yet been localized with certainty in the total thylakoid polypeptide pattern. Thus it is not known if changes in chlorophyll $a$-protein content reflect changes in total polypeptide content.

As noted above, in all the mutants the intensity of protein staining in the region of chlorophyll $a$-protein 1 or of its apoprotein, correlates well with the proportion of the total chlorophyll found in chlorophyll $a$-protein 1 after a brief electrophoresis. The possibility of this chlorophyll-protein existing in vivo but being especially unstable in detergent under our conditions cannot, however, be entirely dismissed. Absence of chlorophyll $a$-protein 1, which is enriched in long wave-length species of chlorophyll, should be reflected in the absorbance spectrum of thylakoids prior to their solubilization with detergent. This situation can be investigated by measuring the difference spectrum of wild-type minus mutant chloroplasts after normalization to a particular wave-length. In our study we normalized the spectra of both wild-type and mutant chloroplasts to an absor- bance of 1 at the wave-length of maximum absorbance and the resulting difference spectra are shown in Figures 2 to 4 . When compared to wild-type thylakoids, those of mutants viridis$z b^{63},-n^{34}$ and $-h^{15}$ (data not shown) as well as viridis- $q^{42}$ are clearly deficient in forms of chlorophyll $a$ absorbing at wave-lengths of 692 $\mathrm{nm}$ and beyond. Low temperature difference spectra show a peak at $690 \mathrm{~nm}$ with a pronounced shoulder around $707 \mathrm{~nm}$. Mutant viridis $-z b^{63}$ is, as previously noted, also deficient in chlorophyll $b$ and chlorophyll $a / b$-protein 2 and this is clearly shown in the difference spectrum (Figure 2) by the peak at $649 \mathrm{~nm}$. Because of arbitrary normalization, differences in light scattering between mutants and overlapping absorbance spectra of all their chlorophyll proteins, it is not possible to use the difference spectra to make quantitative observations as to the proportion of chlorophyll $a$-protein 1 missing from the mutants in vivo. Despite the above uncertainties, we estimate that viridis $-z b^{63}$ has only $80 \%$ of the absorbance at $692 \mathrm{~nm}$ possessed by the wild-type, while viridis $-n^{34}$ has $90 \%$. This result is consistent with the partial loss of chlorophyll $a$-protein 1 from viridis $\cdot n^{34}$ thylakoids compared to the complete loss from viridis $-z b^{63}$. We conclude from the difference spectra that the absence or reduction of chlorophyll $a$-protein 1 from detergent solubilized thylakoids does not result from an altered stability but reflects the in vivo loss of a major chlorophyll $a$ species absorbing preferentially at wave-lengths greater than $692 \mathrm{~nm}$.

Although mutant viridis $-q^{42}$ was selected as a lethal mutant with a possible lesion in photosystem I, the partial rates of electron transport measured do not substantiate this and neither is there any major deficency in chlorophyll $a$ protein 1 . Difference spectra of wild-type minus viridis- $q^{42}$ (Figure 4) indicate that this mutant is also deficient in long wave-length forms of chlorophyll $a$ although the spectra are not identical $(690 \mathrm{~nm}$ peak vs $692 \mathrm{~nm}$ peak at room temperature) with those of wild-type minus mutants viridis $-h^{15},-z b^{63}$ and $-n^{34}$. This was confirmed by recording the difference spectrum of mutant viridis- $q^{42}$ minus mutant viridis- $z b^{63}$ (Figure 5). Viridis $-q^{42}$ contains more of a chlorophyll species absorbing at $696 \mathrm{~nm}$ than does viridis- $z b^{63}$ but is deficient in a species 
absorbing at $685 \mathrm{~nm}$. A difference spectrum between wild-type and mutant viridis- $q^{42}$ of unresolved chlorophyll-proteins exclusive of chlorophyll $a$-protein 1, showed a maximum at $685 \mathrm{~nm}$ (data not shown). This preliminary result indicates that viridis $-q^{42}$ may either be partly deficient in chlorophyll $a$-protein 2 and/or 3 or have an altered chlorophyll $a / b$-protein.

\section{DISCUSSION}

The selection of putative photosystem I mutants by means of their fluorescence induction kinetics is based (25) on the presence of an initial fluorescence $\left(F_{0}\right)$, followed by a rise of the fluorescence to a maximum $\left(F_{m}\right)$ mainly due to photosystem II and the lack of a subsequent decrease which, if present, is due to electron transport through photosystem I (12). If the decrease from the maximum to steady state fluorescence $\left(F_{s}\right)$ is substantially less than in the wild-type such mutants have also been designated as potentially deficient in photosystem I (25). Of the six putative photosystem I mutants studied here, only one, viridis $-z b^{63}$, showed a complete absence of photosystem I activity as indicated both by fluorescence induction kinetics (25) and in vitro electron transport measurements. The other five mutants showed photosystem I activities which ranged from $25 \%$ to $90 \%$ of that of the wild-type (xan-q ${ }^{76}>v i r-q^{42}$ $\left.>x a n-q^{75}>v i r-n^{34}>v i r-h^{15}\right)$ and decreases in $\mathrm{F}_{\mathrm{m}}$ to $\mathrm{F}_{\mathrm{s}}$ which were $5 \%$ to $65 \%$ of that of the wild-type (vir- $n^{34}>x a n-q^{76}>v i r-q^{42}>v i r-h^{15}$ $\left.>x a n-q^{75}\right)(25)$. There is therefore no correlation in the ranking of mutants based on activities of photosystem I electron transport partial reactions and those based on the absolute values of the fluorescence decrease. Furthermore, two of the mutants, xantha- $q^{75}$ and $-q^{76}$ must, on the basis of the results reported here, be more properly described as photosystem II mutants since this was more strongly inhibited than was photosystem I. The two allelic mutants xantha$q^{75}$ and $-q^{76}$ had the lowest variable fluorescence of the six mutants but still $50 \%$ of that in the wild-type. This compares with a photosystem II electron transport activity of less than $10 \%$ of that in the wild-type. Mutants can therefore not be designated as photosystem I mutants solely from the extent of the decrease from $F_{m}$ to $F_{s}$ or from the absence of biphasic variable fluorescence. The small decrease in $F_{m}$ to $F_{s}$ observed in mutants xantha- $q^{75}$ and $-q^{76}$ may be ascribed to the deficiency found in their cytochrome $f$ content in addition to that found in $P 700$ and chlorophyll $a$-protein 1 .

The mutants viridis- $h^{15}$ and $-z b^{63}$ had extremely low $P 700$ contents and the lowest photosystem I rates together with the complete absence of chlorophyll $a$-protein 1. However, there was no such clear correlation in any of the other mutants. This situation may be similar to that in Chlamydomonas during re-greening following treatment with chloramphenicol (6) and in etiolated barley greened in the presence of lincomycin (17) where chlorophyll a-protein I was reduced more than was the photosystem I activity expressed on a chlorophyll basis (18). It has also been demonstrated during greening of etiolated seedlings that photosystem I electron transport develops more rapidly than does measurable $P 700$ activity or formation of stable chlorophyll $a$-protein $1(1,14)$. This situation might be further explored with the isolation of chlorophyll $a$-protein 1 or photosystem I particles from the mutant viridis $-n^{34}$ in which the $P 700$ content is apparently more reduced than is either photosystem I activity or chlorophyll $a$ protein 1 content. The values for $P 700$ content and photosystem I activity in viridis $-n^{34}$ here reported are relatively higher than previously observed (24). The variability of the results may be explained by the use of seed material from a different harvest in the present study

It is generally considered that the long wavelength fluorescence emission at low temperature arises from chlorophyll species associated with photosystem I (11, 12) and more specifically with chlorophyll $a$-protein 1 (2). The evidence for this is the fluorescence emission of digitonin and Triton photosystem I particles and of isolated chlorophyll $a$-protein 1 obtained from gels (2). The results with the mutants studied here do not, however, support such a simple assignment since leaves of viridis $-z b^{63}$ and $-h^{15}$, which do not have any chlorophyll $a$-protein 1 , retain a high ratio of long wavelength fluorescence which differs from the wild-type only in a $6 \mathrm{~nm}$ blue shift of the emission maximum (Figure 6). It is conceivable that the long wavelength fluorescence emission at $77 \mathrm{~K}$ arises from 


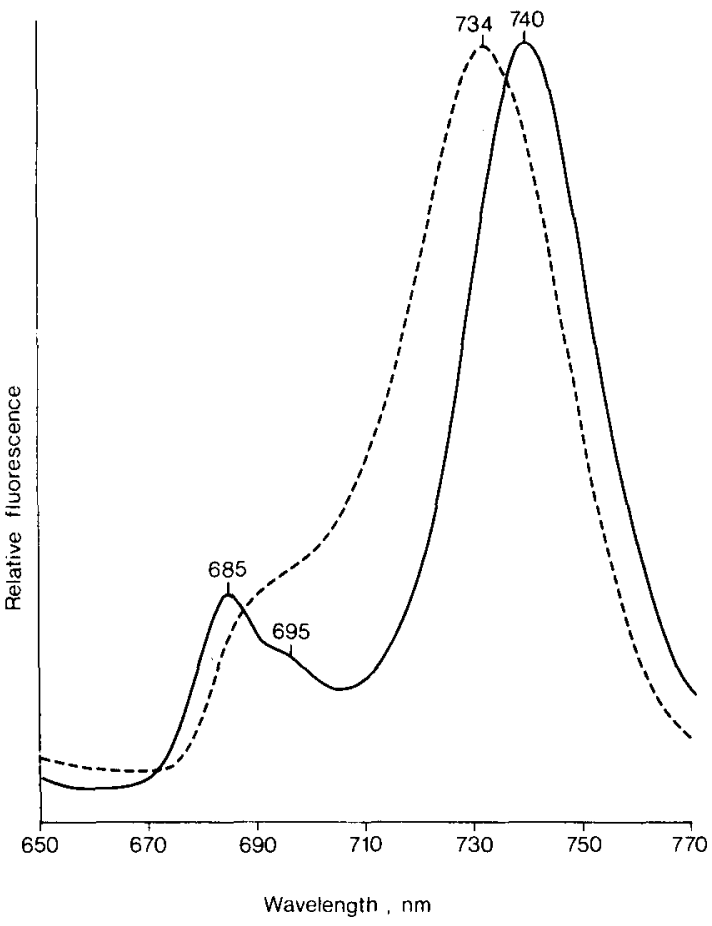

Figure 6. Low temperature $(77 \mathrm{~K})$ fluorescence emission spectra of leaves from wild-type (-) and viridis-zb63(......).

an outer part of chlorophyll $a$-protein 1 whose activity is retained in vivo but lost on solubilization by SDS. An alternative explanation is that the long wave-length fluorescence has multiple origins and that a minor component of shorter emission wave-length in the wild-type becomes predominant when chlorophyll $a$-protein 1 is lost due to mutation.

Although absence of chlorophyll $a$-protein 1 on SDS-PAGE might be the result of decreased stability to SDS-treatment, the difference spectra of wild-type minus mutants viridis $-h^{15},-z b^{63}$ and $-n^{34}$, show a marked reduction in long wavelength forms of chlorophyll $a$. A similar loss of chlorophyll $a_{690}$ and $a_{700}$ has been reported for Chlamydomonas mutants which lack chlorophyll $a$-protein 1 and photosystem I activity (8). From a study of photosystem I particles containing variable chlorophyll/P700 ratios, Mullet et al. (23) concluded that the outer antennae of photosystem I absorbed preferentially at 683 and $701 \mathrm{~nm}$ and the inner at 685 to $690 \mathrm{~nm}$ and $696 \mathrm{~nm}$. If this is correct our results would suggest that only the core antennae of photosystem I together with the reaction centre are lost from the mutants viridis $-h^{15},-z b^{63}$ and $-n^{34}$. This may be contrasted with the restriction of chlorophyll $a$-protein 1 formation by greening leaves in the presence of lincomycin where the difference spectra indicated a removal of chlorophyll species absorbing predominantly at 683 , 692 and $705 \mathrm{~nm}$ although in this case photosystem I activity was unimpaired (17).

The loss of chlorophyll $a$-protein 1 and its apoprotein is accompanied in all mutants with a similar reduction in the content of three polypeptides with molecular weights of $18,300,15,200$ and 13,500. Two of these polypeptides incorporate ${ }^{59} \mathrm{Fe}$ (unpublished data) and have consequently been assigned to the iron-sulfur centres shown by low temperature ESR to be an integral part of photosystem I (13). The chloroplast photosystem I reaction centre $(P 700)$ appears to form an integral complex with several polypeptides of low molecular weight (7). The extent to which this complex can be assembled in the absence of any one of its components is uncertain. If all are required for the proper synthesis and assembly, then there are likely to be several non-allelic viridis mutants which lack a functional photosystem I. In addition, mutants lacking polypeptides necessary for the correct assembly of the complex are expected. These may well have pleiotropic effects which result in the partial loss of components somewhat removed from the photosystem I reaction centre.

Among the mutations studied here, only that in viridis $-n^{34}$ produces a lesion which is confined to the integral components of photosystem I, that is, chlorophyll $a$-protein $1, P-700$, and associated iron-sulfur proteins. Mutants viridis- $h^{15}$ and $-z b^{63}$ are also partly deficient in chlorophyll $a / b$ protein 2 and cytochrome $f$ and may represent assembly mutants. Mutant viridis $-z b^{63}$ should be the most useful for further studies on photosystem II electron transport. This mutant is not only completely lacking photosystem I activity but the deficiency in chlorophyll $a$-protein 1 and additionally in chlorophyll $a / b$-protein 2 results in an enrichment of photosystem II components which is comparable with that in photosystem II particles prepared by fractionation with digitonin.

Specific deficiency in chlorophyll $a$-protein I 
could be caused by mutation in three nuclear genes of barley. It may also be noted that mutations in chloroplast DNA can lead to loss of chlorophyll $a$-protein $1(8,15)$. It is evident that this type of photosystem I mutants deficient in chlorophyll $a$-protein 1 can only be useful in further resolving these complex structural interrelationships if in vitro protein synthesis from RNA is investigated. An alternative approach is the search for higher plant mutants which are compositionally indistinguishable from the wildtype but which have a functionally incompetent photosystem I.

\section{ACKNOWLEDGEMENTS}

We wish to thank Professor D. vON WETtSTEIN for a critical review of the manuscript. Financial support was provided by the Commission of European Communities contract 029-76ES DK solar energy program to Professor D. VON WETTSTEIN.

\section{REFERENCES}

1. Acker, S., A. Picaud \& J. Duranton: Des activitiés photosynthétiques sans les complexes pigmentaires CP1 et CP2. Biochim. Biophys. Acta 440, 269-277 (1976)

2. Anderson, J. M., J. C. Waldron \& S. W. THORNE: Chlorophyll-protein complexes of spinach and barley thylakoids. Spectral characterization of six complexes resolved by an improved electrophoretic procedure. FEBS Letters, 92, 227-233 (1978)

3. Anderson, J. M., K. C. Woo \& N. K. BoardMAN: Photochemical systems in mesophyll and bundle sheath chloroplasts of $\mathrm{C}_{4}$ plants. Biochim. Biophys. Acta 245, 398-408 (1971)

4. APEL, K. \& K. KLOPPSTECH: The plastid membranes of barley (Hordeum vulgare). Light-induced appearance of mRNA coding for the apoprotein of the light-harvesting chlorophyll $a / b$ protein. Eur. J. Biochem. 85, 581-588 (1978)

5. ARnon, D. I.: Copper enzymes in isolated chloroplasts. Polyphenoloxidase in Beta vulgaris. Plant Physiol. 24, 1-14 (1949)

6. Bar-Nun, S., R. Schantz \& I. Ohad: Appearance and composition of chlorophyll-protein complexes I and II during chloroplast membrane biogenesis in Chlamydonomas reinhardi $y-1$. Biochim. Biophys. Acta 459, 451-467 (1977)
7. Bengis, C. \& N. Nelson: Subunit structure of chloroplast photosystem I reaction center. J. Biol. Chem. 252, 4564-4569 (1977)

8. Bennoun, P., J. Girard \& N.-H. Chua: A uniparental mutant of Chlamydomonas reinhard. tii deficient in the chlorophyll-protein complex CP I. Molec. gen. Genet. 153, 343-348 (1977)

9. Boardman, N. K. \& J. M. Anderson: Fractionation of the photochemical systems of photosynthesis. II. Cytochrome and carotenoid contents of particles isolated from spinach chloroplasts. Biochim. Biophys. Acta 143, 187-203 (1967)

10. Chua, N.-H., K. Matun \& P. Bennoun: A chlorophyll-protein complex lacking in photosystem I mutants of Chlamydomonas reinhardtii. J. Cell Biol. 67, 361-377 (1975)

11. Evans, M. C. W.: Electron paramagnetic resonance studies in photosynthesis. In: Primary Processes of Photosynthesis (ed. J. Barber) Elsevier/North-Holland Biomedical Press, Amsterdam, pp. 433-464 (1977)

12. GovindjeE, \& R. GovindjeE: Introduction to photosynthesis. In: Bioenergetics of Photosynthesis (ed. Govindjee) pp. 1-50. Academic Press. New York, San Francisco and London (1975)

13. Heathcote, P., D. L. Williams-Smith, C. K. Simra \& M. C. W. Evans: The role of the membrane-bound iron-sulfur centres $\mathrm{A}$ and $\mathrm{B}$ in the photosystem I reaction centre of spinach chloroplasts. Biochim. Biophys. Acta 503, 333342 (1978)

14. Henningsen, K. W. \& N. K. Boardman: Development of photochemical activity and the appearance of the high potential form of cytochrome $b-559$ in greening barley seedlings. Plant Physiol. 51, 1117-1126 (1973)

15. HerrmanN, F.: Genetic control of pigment protein complexes I and la of the plastid mutant en:alba-1 of Antirrhinum majus. FEBS Lett. 19. 267-269 (1971)

16. Hiller, R. G., T. B. G. Pilger \& D. Campbell: Extraction and stabilisation of the dimer of chlorophyll-protein complex II. Plant Science Letters 14, 7-11 (1979)

17. Hiller, R. G., T. B. G. Pillger \& S. Genge: Effect of lincomycin on the chlorophyll protein complex I content and photosystem I activity of greening leaves. Biochim. Biophys. Acta 460, 431-444 (1977)

18. Hiyama, T. \& B. Ke: Difference spectra and extinction coefficients of P700. Biochim. Biophys. Acta 267, 160-171 (1972)

19. Høyer-Hansen, G. \& D. J. Simpson: Changes in the polypeptide composition of internal membranes of barley plastids during greening. Carlsberg Res. Commun. 42, 379-389 (1977) 
20. Machold, O., D. J. Simpson \& B. L. Møller: Chlorophyll-proteins of thylakoids from wildtype and mutants of barley (Hordeum vulgare L.) Carlsberg Res. Commun. 44, 235-254 (1979)

21. Miles, C. D., J. P. Markwell \& J. P. THORNBER: Effect of nuclear mutation in maize on photosynthetic activity and content of chlorophyll-protein complexes. Plant Physiol. 64, 690-694 (1979)

22. MiLl.ER, K. R.: A chloroplast membrane lacking photosystem I changes in unstacked membrane regions. Biochim. Biophys. Acta 592, 143-152 (1980)

23. Mullet, J. E., J. J. Burke \& C. J. Arntzen: Chlorophyll proteins of photosystem I. Plant Physiol. 65, 814-822 (1980)

24. Mølier, B. L., R. M. Smillie \& G. HøyerHansen: A photosystem I mutant in barley (Hordeum vulgare L.). Carlsberg Res. Commun. 45 , $87-99(1980)$
25. Simpson, D. J. \& D. von Wettstein: Macromolecular physiology of plastids. XIV. Viridis mutants in barley: Genetic, fluoroscopic, and ultrastructural characterization. Carlsberg Res. Commun. 45, 283-314 (1980)

26. Wettstein, D. von \& K. KRistiansen: Stock list for nuclear gene mutants affecting the chloroplast. Barley Genet. Newslett. 3, 113-117 (1973)

27. Wettstein, D. von, A. KahN, O. F. Nielsen \& S. Gough: Genetic regulation of chlorophyll synthesis analysed with mutants in barley. Science 184, 800-802 (1974)

28. Wettstein, D. von, B. L. Møller, G. HøyerHANSEN \& D. Simpson: Mutants in the analysis of the photosynthetic membrane polypeptides. In: Origin of chloroplasts (J. A. Schiff \& R. Y. Stanier, eds.) Elsevier/North-Holland, Biomedical Press, Amsterdam, in press (1980) 